14

\title{
Имитационное моделирование эпилептиформной активности сетью нейроподобных радиотехнических осцилляторов
}

\author{
(C) Н.М. Егоров, ${ }^{1,2}$ В.И. Пономаренко, ${ }^{1,3}$ И.В. Сысоев, ${ }^{1,3}$ М.В. Сысоева ${ }^{1,2}$ \\ ${ }^{1}$ Саратовский филлиал Института радиотехники и электроники им. А. Котельникова РАН, \\ 410019 Саратов, Россия \\ ${ }^{2}$ Саратовский государственный технический университет им. Ю.А. Гагарина, \\ 410054 Саратов, Россия \\ ${ }^{3}$ Саратовский национальный исследовательский государственный университет им. Н.Г. Чернышевского, \\ 410012 Саратов, Россия \\ e-mail: egorov.n.m.omnis@gmail.com
}

Поступило в Редакцию 30 июля 2020 г.

В окончательной редакции 31 августа 2020 г.

Принято к публикации 14 сентября 2020 г.

Разработана радиотехническая схема иерархически организованной таламо-кортикальной сети мозга с внешним входом. Проведено исследование полученной модели, в которой обнаружены различные режимы вынужденных и автоколебаний, включая режим с жестким возбуждением. Обнаружено, при каких параметрах схема демонстрирует активность, сходную с пик-волновыми разрядами при абсансной эпилепсии, реализуя гипотезу, что пик-волновой разряд является длительным переходным процессом вблизи бифуркации возникновения цикла из сгущения фазовых траекторий.

Ключевые слова: имитационное моделирование, модель ФитцХью-Нагумо, таламо-кортикальная сеть мозга, эпилептиформная активность.

DOI: 10.21883/JTF.2021.03.50532.237-20

\section{Введение}

К настоящему времени показано, что сложные сетевые взаимодействия могут обусловливать функционирование самых различных природных и техногенных объектов, а изменения в сетях - приводить к переходам от нормальной динамики к патологической $[1,2]$. Эпилепсия представляет собой одно из самых распространенных и социально значимых заболеваний, в значительной степени обусловленное патологическими структурно-функциональными взаимодействиями внутри коры, а также между корой и глубокими структурами мозга, прежде всего таламусом [3] и гиппокампом [4]. Изучение большинства форм эпилепсии в настоящее время основывается на анализе экспериментальных данных электромагнитной активности мозга и дальнейшем воспроизведении полученных эффектов в моделях.

Моделирование электромагнитной активности мозга - фундаментальная междисциплинарная проблема, для решения которой в настоящее время задействованы значительные силы (Blue Brain Project, The Human Brain Project и др.), но заявленные цели пока не достигнуты, в том числе потому, что ставилась цель моделирования всего мозга в целом, а не отдельных его структур или режимов функционирования. Ряд работ показывает, что можно рассчитывать на успех, если ограничиться определенным типом патологии и рассматривать только области и структуры мозга, непосредственно вовлеченные в ее формирование [5-7]. Такие математические модели воспроизводят большое число наблюдае- мых характеристик экспериментальных сигналов, включая спектр, амплитуду, форму колебаний и механизмы переключения, а также хорошо масштабируются, но имеют два важных недостатка из-за большого числа модельных нейронов в них (обычно не менее 500). Во-первых, механизмы формирования патологической активности на них можно изучать только статистически. Во-вторых, реализация таких моделей в натурном эксперименте (биологическом в культурах клеток или радиотехническом в виде аналоговой схемы) затруднена. Между тем для верификации моделей и их дальнейшего применения, например, для разработки и тестирования лечебных воздействий (сейчас апробация производится на животных моделях $[8,9])$ было бы крайне желательно повторить полученные в математической модели результаты в радиотехническом эксперименте, так как только в натурном эксперименте естественным образом возникают шумы, неидентичность и неидеальность элементов сети, характерные для изучаемой системы. Радиотехнический эксперимент проще биологического, а первым шагом к его экспериментальной реализации является имитационное моделирование.

Чтобы разработать радиотехническую установку, необходимо использовать более простую модель, состоящую из меньшего числа элементов, но при этом сохраняющую основные сетевые характеристики предложенных в [5-7] моделей и реализуемую в виде экспериментальной схемы. Целью настоящей работы является реализация иерархически организованной сетевой модели части таламо-кортикальной сети мозга, ответственной 
за абсансные приступы, в виде имитационной радиотехнической схемы. Такая схема будет создана впервые. Схема радиотехнической установки разрабатывается на компьютере с использованием моделей реальных электронных компонентов. Это позволяет существенно сократить и упростить дальнейшую разработку и отладку экспериментальной установки „в железе“ за счет следующего. Во-первых, будет готов и оттестирован макет, включающий все радиотехнические компоненты и их соединения, способный воспроизводить требуемую эпилептиформную активность. Во-вторых, будет частично учтено влияние неидеальности отдельных компонентов на работу схемы. В-третьих, будет упрощено перепроектирование схемы, если в эксперименте полученные в модели режимы не будут воспроизводиться, например, из-за отсутствия некоторых компонентов или изменения их свойств относительно номинала. Предлагаемая схема должна состоять из относительно малого числа модельных нейронов, но повторять принципы организации, заложенные в математическую модель [7], так что дальнейшая аппаратная реализация не будет затруднительна.

\section{1. Модель}

\section{1. Математическая модель эпилептиформной активности}

В работе [7] была предложена стохастическая мезомасштабная модель эпилептиформной активности, состоящая из 172 модельных нейронов (80 кортикальных, 60 таламических и 32 периферических); „стохастическая“, так как в динамику отдельного нейрона добавлялся аддитивный динамический шум, „мезомасштабная“ в соответствии с идеями работы [10], где предложено не пытаться моделировать каждый нейрон и каждую нейронную связь, а добиваться, чтобы отдельные уравнения моделировали мезомасштабную электрическую динамику в мозге. То есть вместо динамики мембранного потенциала сомы и аксона каждого отдельного нейрона, мезомасштабная модель рассматривает пространственное среднее потенциала популяции нейронов, причем таких популяций учитывается множество для каждого типа клеток и каждой структуры (десятки или сотни, как в [11], где рассмотрена модель из 656 нейронов), в отличие от популяционных моделей для нейронных сетей, где одно уравнение или небольшой набор уравнений относится ко всей популяции $[12,13]$. Связи между отдельными популяциями и количество таких популяций следуют известным фактам из анатомии головного мозга (см. [14] для крыс-моделей). Основной идеей создания такой модели было предположение о том, что главную роль в генерации высоко синхронизованной активности нейронов коры и таламуса играет именно структура сети, т. е. наличие в ней патологических петель обратной связи, возникших случайно или закрепленных генетически в популяции $[15,16]$.

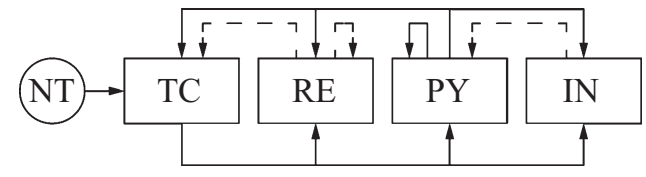

Рис. 1. Структурная схема ансамбля нейронов. Сплошные линии - положительные связи, штриховые линии - отрицательные связи.

Основными недостатками модели [7] можно назвать отсутствие деления модельных „нейронов“ на возбуждающие и тормозные (все связи являются положительными) и наличие шума в модели каждого индивидуального нейрона. В работе [17] эти недостатки был исправлены, при этом основную идею и структуру модели оставили без изменений. Эту модель назвали динамической мезомасштабной моделью. Схема модели была получена в результате обобщения работ $[12,18]$. В модели [17] каждый отдельный нейрон моделирует собой группу близких нейронов: РY - пирамиды (200 нейронов), ТС — таламокортикальные клетки (120 нейронов), IN - интернейроны (50 нейронов) и RE - ретикулярные нейроны (120 нейронов). На рис. 1 приведена схема, иллюстрирующая архитектуру связей в предложенной модели. Интегральный сигнал нейронов, входящих в популяции РY и IN, является аналогом сигнала локальных потенциалов (LFP) первичной соматосенсорной коры (SI), TC представляет собой совокупность клеток вентропостериального медиального ядра таламуса (VPM), a RE - клеток ретикулярного ядра таламуса (RTN). Связи, исходящие из РY и ТC, являются коллатеральными (это означает, что если из РY выходит сигнал, то он обязательно должен прийти и в RE, и в ТС; сигнал из ТC приходит в $\mathrm{RE}$, IN и PY). Кроме того, в модель были включены нейроны тройничного нерва (nervus trigeminus, NT), поскольку известно, что эпилепсия может возникать в ответ на внешний стимул [19]. В настоящей работе количество нейронов было пропорционально сокращено до минимально возможного: $N_{\mathrm{PY}}=4, N_{\mathrm{IN}}=1, N_{\mathrm{TC}}=4$, $N_{\mathrm{RE}}=4, N_{\mathrm{NT}}=1$.

\section{2. Принципиальная схема одного нейрона}

Для моделирования нейронов каждого типа в работах $[7,17]$ были использованы уравнения ФицХью-Нагумо в виде [20]. В настоящей работе в качестве элементов сети использовались системы ФицХью-Нагумо, каждая из которых описывается следующим модельным уравнением (1), как это было предложено в [21]:

$$
\begin{aligned}
& \varepsilon \dot{u}(t)=u(t)-\frac{u^{3}(t)}{3}-v(t), \\
& \dot{v}(t)=u(t)+a,
\end{aligned}
$$

где $u(t)$ и $v(t)$ - переменные, качественно соответствующие трансмембранному напряжению и переменной 


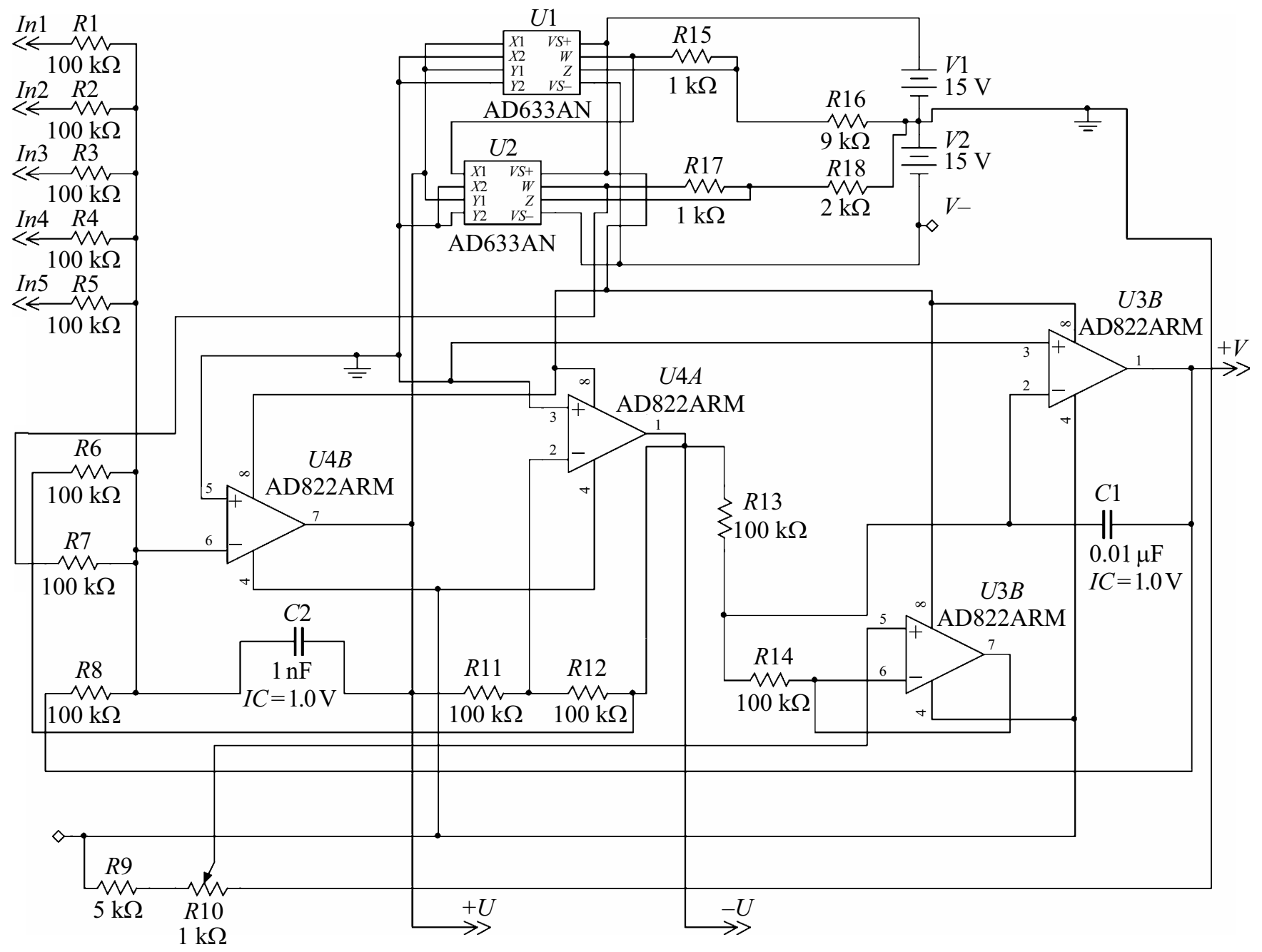

Рис. 2. Принципиальная схема одного нейрона.

активации ионного тока соответственно (будем также называть их активатором и ингибитором соответственно); $\varepsilon$ - параметр соотношения временных масштабов, характеризующий относительную скорость активации (деактивации) ионного тока; $a-$ пороговый параметр системы, который может принимать значения из отрезка $a \in[0 ; 2]$. Эта модель удобна наличием одного управляющего параметра, малой по сравнению, например, с моделью Хиндмарш-Роуз размерностью и относительно простой по сравнению, например, с моделью Моррис-Лекара нелинейностью (отсутствие гиперболических функций), что упрощает реализацию радиотехнической модели и анализ полученных результатов (фактически это простейшая из известных двумерных моделей нейронов [22]).

Для математической модели уединенного нейрона и радиотехнической схемы, составленной из идеальных элементов, значениям $a>1$ соответствует возбудимый режим, при котором траектории системы стремятся к устойчивой точке, а значениям $a<1-$ автоколебательная динамика (устойчивый предельный цикл на фазо- вой плоскости), возникающая через суперкритическую бифуркацию Андронова-Хопфа при критическом значении $a_{c r}=1$. Для радиотехнической схемы отдельного нейрона, включающей в себя неидеальные операционные усилители и умножители, нелинейная кубическая функция аппроксимируется неточно, поэтому бифуркационное значение параметра смещается и автоколебательная динамика наблюдается при $a \leq 1.025$. Для связанных нейронов переход к колебательному режиму возможен при еще больших значениях параметра $a$.

На рис. 2 представлена принципиальная схема одного нейрона, разработанная в [23] на основе математической модели, предложенной в [21], и используемая в нашей системе. Схема содержит два аналоговых умножителя $U 1$ и $U 2$ и два сдвоенных операционных усилителя $U 3, U 4$. Элементы $U 4 B$ и $U 3 A$ выполняют функцию интеграторов. Их выходные напряжения обозначены как $+U$ и $+V$ соответственно (они соответствуют переменным $u, v$ в уравнении (2)). На элементе $U 4 A$ реализован инвертирующий усилитель с коэффициентом усиления -1 , на его выходе мы имеем $-U$. Кубическое 

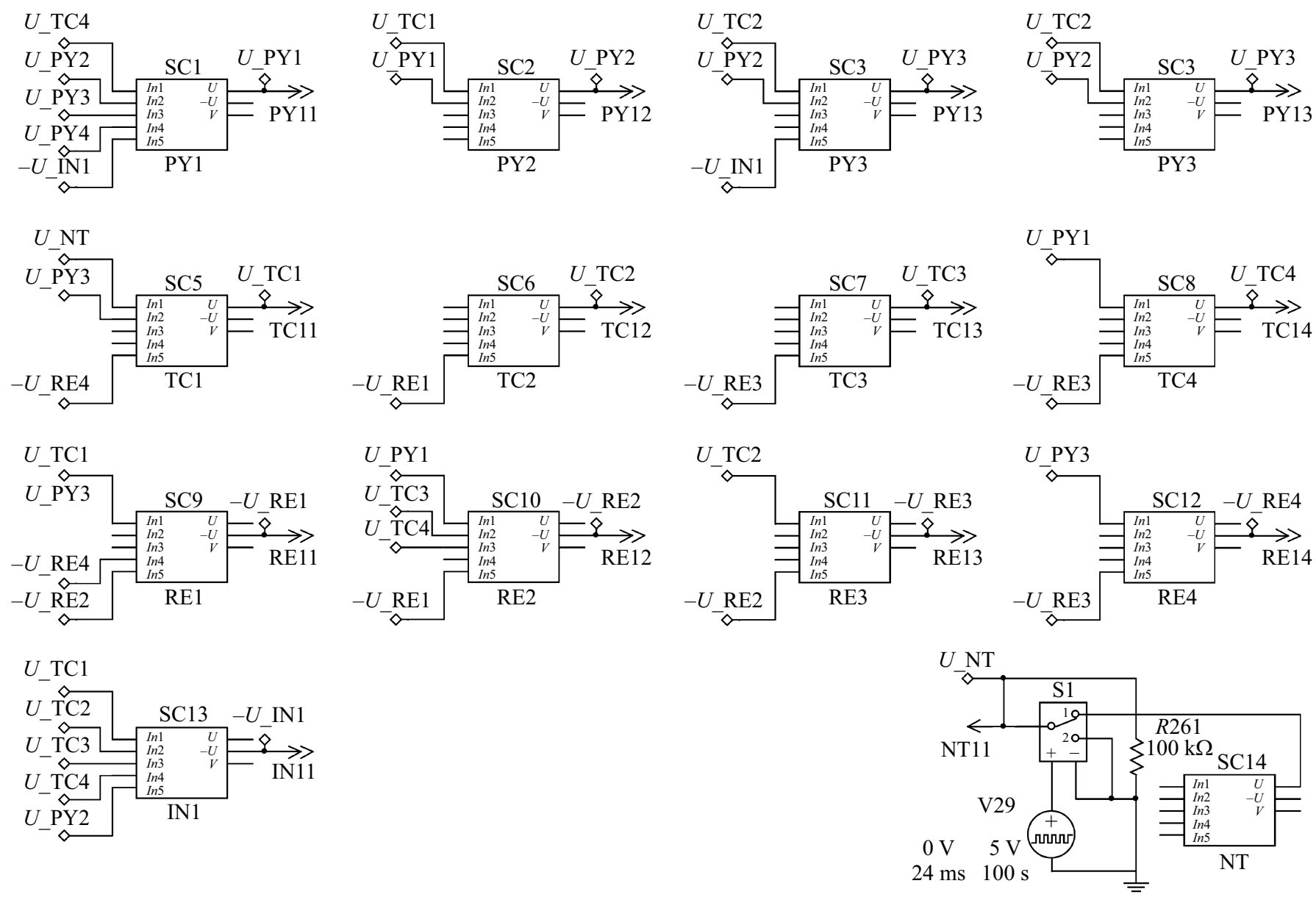

Рис. 3. Принципиальная схема таламо-кортикальной сети с внешним входом.

преобразование выполняется умножителями. Повторитель $U 3 B$ предназначен для подключения к схеме потенциометра $R 10$, напряжение на среднем выводе которого эквивалентно $A$. Входы $\operatorname{In} 1-\operatorname{In} 5$ принимают воздействия от других нейронов. Резисторы $R_{i n}=R 1-R 5$ отвечают за силу связи $k$ между нейронами, которая рассчитывается через соотношение (2):

$$
k=\frac{R_{6}}{R_{\text {in }}} .
$$

Параметр шкалы времени принимает значение $\varepsilon_{e x}=R 7 \cdot C 2$. Динамика рассматриваемого осциллятора описывается безразмерными уравнениями (1), в которых

$$
u=\frac{U}{u^{\prime}}, \quad v=\frac{V}{v^{\prime}}, a=\frac{A}{a^{\prime}}, t=\frac{T}{t^{\prime}} \text { и } \varepsilon=\frac{\varepsilon_{e x}}{t^{\prime}},
$$

где

$$
u^{\prime}=1 \mathrm{~V}, v^{\prime}=1 \mathrm{~V}, a^{\prime}=1 \mathrm{~V}, t^{\prime}=R 13 \cdot C 1=1 \mathrm{~ms} .
$$

Экспериментальные напряжения $U, V$ и $A$ измеряются в вольтах, а время в эксперименте измеряется в миллисекундах.
Параметр $a$ зависит от номинала потенциометра $R 10$ (рис. 2) и влияет на возможность возбуждения колебаний. Этот параметр можно найти, зная $A-$ величину, выраженную в процентах на потенциометре. С учетом соотношения номиналов резисторов $R 9$ и $R 10$, формула для пересчета $A$ в $a$ выглядит следующим образом:

$$
a=2.5\left(1-\frac{A}{100 \%}\right) \text {. }
$$

\section{3. Принципиальная схема таламо-кортикальной сети с внешним входом}

На базе программного обеспечения National Instruments Multisim был построен ансамбль из 14 осцилляторов ФитцХью-Нагумо (включая аналог тройничного нерва - внешнего входа). Принципиальная схема всей сети показана на рис. 3. На таламокортикальный нейрон (ТC) подается внешнее воздействие от тройничного нерва (NT), далее таламокортикальные нейроны оказывают влияние положительной обратной связью $(+U$, рис. 2$)$ на все остальные группы нейронов кроме себя самих. В свою очередь, пирамидальные 
Таблица 1. Расстройка по параметру $a_{b r}$ (множитель $10^{-3}$ )

\begin{tabular}{c|c|c|c|c|c|c|c|c|c|c|c|c}
\hline PY1 & PY2 & PY3 & PY4 & RE1 & RE2 & RE3 & RE4 & TC1 & TC2 & TC3 & TC4 & IN \\
\hline-0.025 & 0.025 & -0.1 & 0.1 & 0.1 & -0.075 & 0 & -0.025 & 0 & -0.05 & -0.025 & 0.075 & 0
\end{tabular}

нейроны (PY) также действуют на все группы нейронов, включая себя. Интернейрон же воздействует на пирамидальный нейрон, но с отрицательной по знаку связью $(-U$, рис. 2). Также используют отрицательную связь и ингибиторные нейроны (RE), влияя на таламокортикальные нейроны и на самих себя. Воздействие на систему, оказываемое осциллятором, выполняющим роль тройничного нерва (NT), поступает с положительной по знаку связью на один из входов In нейрона ТC1.

Схема реализует простую линейную связь между генераторами, имитирующую электрическую синаптическую связь между нейронами, это соединение соответствует соединению двух электронных генераторов через резистор:

$$
\begin{aligned}
& \varepsilon \dot{u}_{i}(t)=u_{i}(t)-\frac{u_{i}^{3}(t)}{3}-v_{i}(t)+\sum_{j} k_{i j} u_{j}, \\
& \dot{v}_{i}(t)=u_{i}(t)+a_{i}
\end{aligned}
$$

Модуль коэффициента связи $\left|k_{i j}\right|$, рассчитанный по формуле (2), внутри таламо-кортикальной сети для присутствующих связей обозначим $k_{b r}$ (нижний индекс $b r$ означает brain, таламо-кортикальная сеть мозга), а коэффициент связи от внешнего входа $-k_{\mathrm{NT}}$ (нижний индекс NT означает nervus trigeminus, тройничный нерв, внешний вход сети). одинаковый для всех нейронов таламо-кортикальной сети в рамках одного опыта.

Аналогично обозначим среднюю величину порогового параметра системы внутри таламо-кортикальной сети $\bar{a}_{b r}$, величину этого параметра для тройничного нерва $-a_{\mathrm{NT}}$. Для описания неидентичности биологических нейронов осцилляторы ФитцХью-Нагумо были незначительно (табл. 1) расстроены по параметру $a_{b r}$. На графиках ниже и в тексте всегда речь будет идти о средней по ансамблю $\bar{a}_{b r}$, расстройка для конкретного нейрона не менялась при изменении $\bar{a}_{b r}$. Параметр $a_{\mathrm{NT}}$ во всех опытах был равен 1.025 .

\section{4. Радиотехническая модель эпилептиформной активности}

В соответствии с [19] считаем, что эпилептический разряд зарождается в ответ на кратковременный импульс, пришедший с тройничного нерва. На схеме таламо-кортикальной сети с внешним входом (рис. 3) видно, что внешнее воздействие от тройничного нерва может быть включено и выключено в произвольные моменты времени за счет ключа $S 1$. Используется ключ, управляемый напряжением (Voltage Controlled SPDT), на него подается положительный прямоугольный импульс с генератора импульсов V29 (длительность импульса составляет $70 \mathrm{~ms}$, период повторения импульса $100 \mathrm{~s}$ ). Когда приходит импульс, ключ открывается и подается воздействие на сеть, когда импульс заканчивается, ключ закрывается и воздействие прерывается.

Нейрон внешнего входа NT должен находиться в колебательном режиме, а вся сеть - в подпороговом режиме. После подачи кратковременного стимула с внешнего входа, сеть должна начать колебаться и продолжить колебаться еще какое-то время после отключения внешнего входа. Именно этот долгий переходной процесс и будем считать эпилептическим разрядом.

\section{2. Результаты}

\section{1. Автономная система связанных нейронов таламо-кортикальной сети}

В пространстве параметров $\left(\bar{a}_{b r}, k_{b r}\right)$ по точкам была построена кривая, соответствующая бифуркации рождения цикла из сгущения фазовых траекторий [24] в отсутствие внешнего воздействия $k_{\mathrm{NT}}=0$ (на рис. 4 обозначена штриховой линией с кружочками). Кривая строилась при $k_{b r}=0, \ldots, 0.6$, при $k_{b r} \geq 0.7$ аттрактор разрушается, амплитуда неограниченно увеличивается. Несвязанные нейроны $\left(k_{b r}=0\right)$ переходят в колебательный режим при $\bar{a}_{b r, c r}=1.025$ (в этом случае $\bar{a}_{b r, c r}>1$ из-за того, что при моделировании схемы использованы модели реальных операционных усилителей и умножителей). С увеличением $k_{b r}$ критическое значение параметра $\bar{a}_{b r, c r}$, при котором происходит переход от возбудимого режима к автоколебательному, монотонно увеличивается, достигая $\bar{a}_{b r, c r}=1.47$ при $k_{b r}=0.6$.

На рис. 5 изображены временные ряды динамики нейрона ТC1 в отсутствие внешнего воздействия (остальные нейроны демонстрировали аналогичное поведение): слева при $\bar{a}_{b r}=1.250$ - выше бифуркационной кривой (рис. 5, $a$ ) в области пространства параметров, где колебания отсутствуют, в центре (рис. 5, $b$ ) при $\bar{a}_{b r}=1.1325$ - почти на линии бифуркации (виден очень длинный переходной процесс), справа при $\bar{a}_{b r}=1.125$ (рис. 5,c) 一 ниже линии бифуркации в области, где при достаточном возмущении быстро устанавливаются колебания большой амплитуды. 

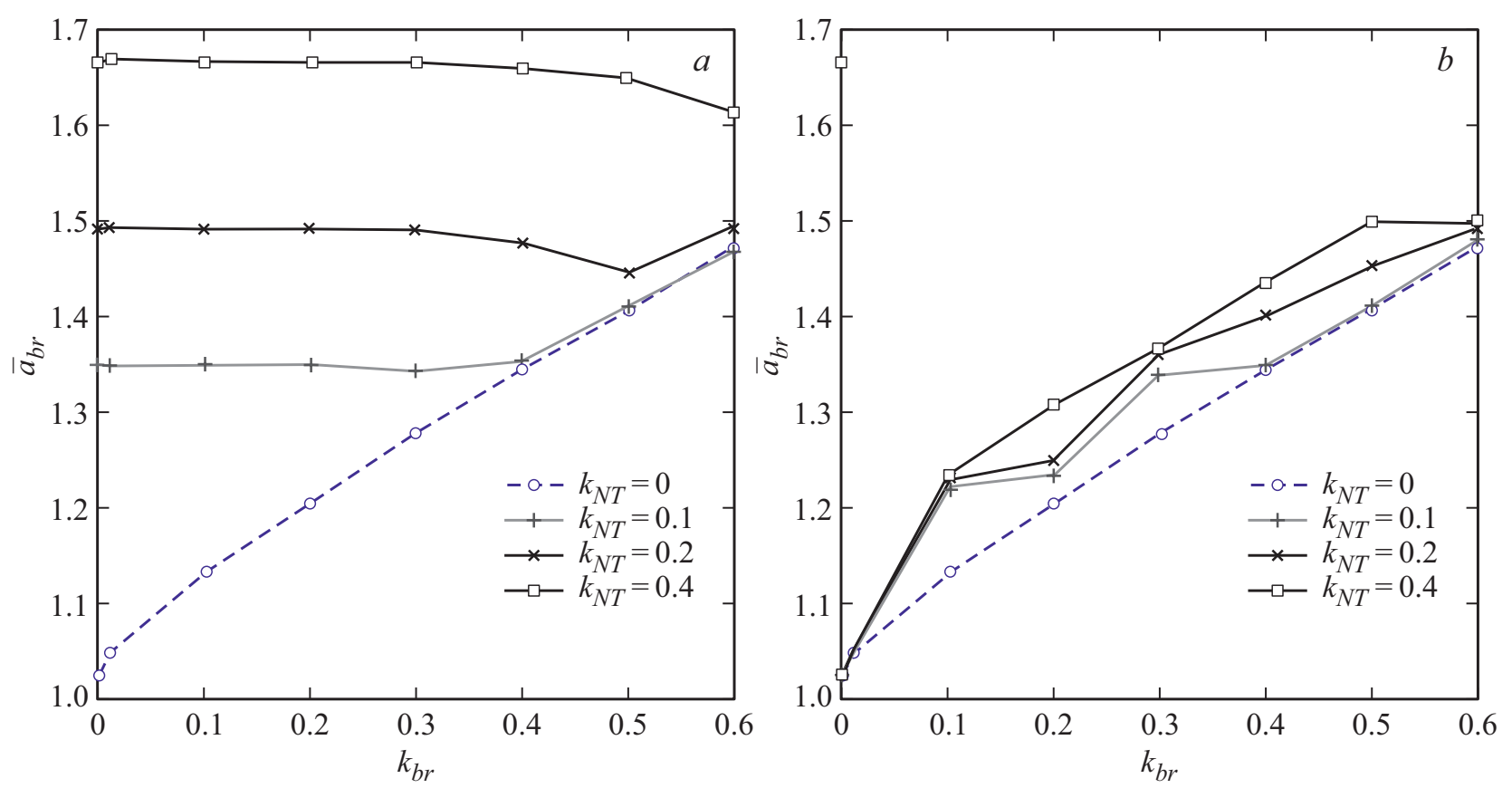

Рис. 4. Семейство кривых на плоскости параметров $\left(\bar{a}_{b r}, k_{b r}\right): a$ - для нейрона ТC1; $b$ - для нейрона ТС3.
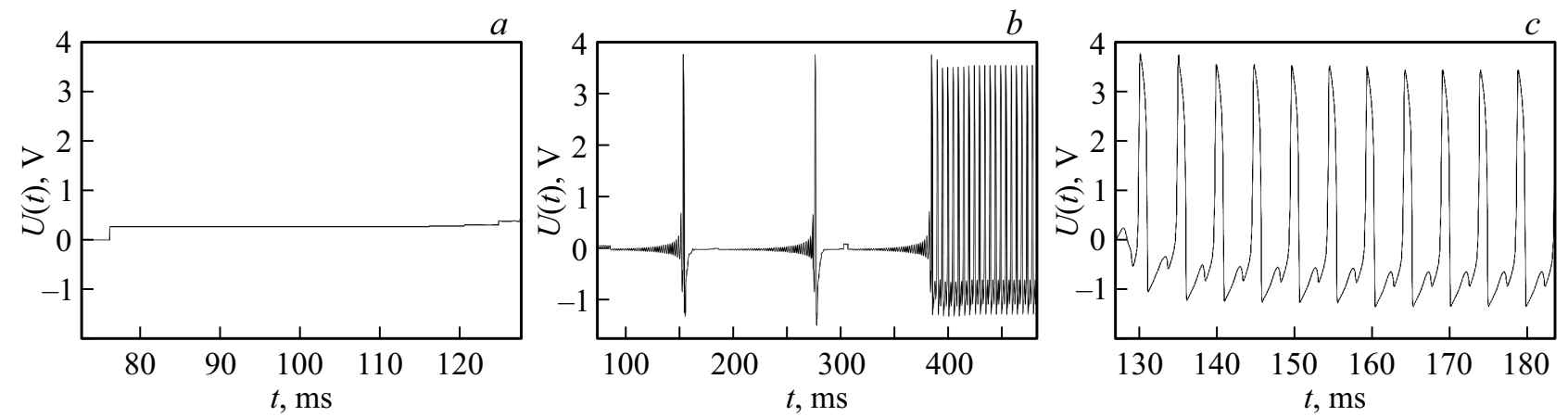

Рис. 5. Временнб́е ряды автономной системы $\left(k_{\mathrm{NT}}=0, k_{b r}=0.1\right): a-$ выше бифуркационной кривой $\bar{a}_{b r}=1.250 ; b-$ вблизи бифуркационной кривой $\bar{a}_{b r}=11325 ; c-$ ниже бифуркационной кривой $\bar{a}_{b r}=1.125$.

\section{2. Неавтономная система связанных нейронов таламо-кортикальной сети}

Внешнее воздействие на исследуемую сеть осуществляется со стороны системы ФитцХью-Нагумо, которая находится в колебательном режиме $\left(a_{\mathrm{NT}}=1.025\right)$ и моделирует влияние тройничного нерва. Воздействие подавалось на нейрон типа ТС (был выбран ТС1) в соответствии с физиологическими представлениями об устройстве периферической нервной системы. Сила воздействия менялась за счет изменения номинала резистора $R 1$ (рис. 2), влияющего на коэффициент связи $k_{\mathrm{NT}}$.

На рис. 4 показаны кривые, соответствующие переходу системы из возбудимого режима в режим резонансных колебаний при разной силе воздействия $k_{\mathrm{NT}}=0.1,0.2,0.4$. Показаны результаты для нейрона сети, на который непосредственно приходит сигнал с внешнего входа, ТС1 (рис. 4,a) и для нейрона сети, который удален от внешнего входа, ТС3 (рис. 4,b). Поскольку нейроны NT и ТC1 существенно отличаются собственными параметрами $a$, они имеют различные частоты собственных колебаний (частота колебаний нейрона внешнего входа NT меньше, чем частота колебаний нейрона ТC1, на который приходит воздействие). Поэтому при малых амплитудах воздействия (малом коэффициенте связи $k_{\mathrm{NT}}$, выше кривой) отдельный нейрон демонстрирует низкоамплитудные подпороговые колебания (рис. 6,a). При достаточном удалении от кривой их амплитуда столь мала, что ее невозможно детектировать имеющимися средствами. Ниже кривой нейрон демонстрирует высокоамплитудные нелинейные вынужденные колебания. С уменьшением $\bar{a}_{b r}$ далее значения, приведенного на кривой, амплитуда вынужденных колебаний не уменьшается, поскольку одновременно с удалением от резонанса происходит увеличение добротности (параметр $\bar{a}_{b r}$ одновременно ответственен за 

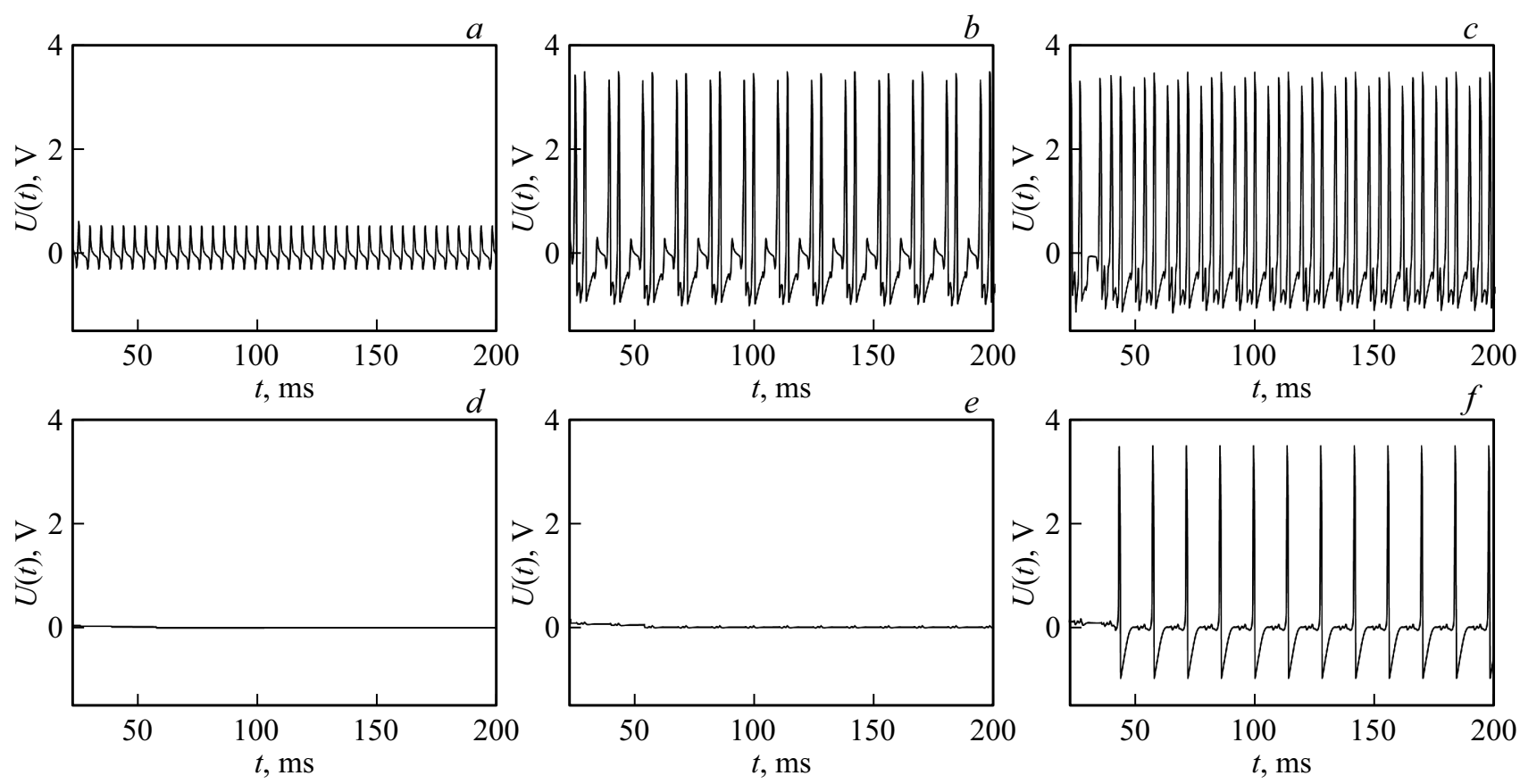

Рис. 6. Временные ряды неавтономной системы из нейрона ТС1 (сверху) и нейрона ТС3 (снизу) при $k_{\mathrm{NT}}=0.2, k_{b r}=0.3: a, d-$ $a_{b r}=1.55 ; b, e-a_{b r}=1.45 ; c, f-a_{b r}=1.35$.

частоту и за коэффициент линейного затухания), затем вынужденные колебания преобразуются в автоколебания, модулированные внешним воздействием.

Для нейрона ТC1 переход в колебательный режим происходит сразу скачком при включении внешнего воздействия: для $k_{\mathrm{NT}}=0.1$ при $\bar{a}_{b r}=1.3475$; для $k_{\mathrm{NT}}=0.2$ при $\bar{a}_{b r}=1.4925$; для $k_{\mathrm{NT}}=0.4$ при $\bar{a}_{b r}=1.6675$. При небольших $k_{b r}$ кривые идут параллельно оси абсцисс, т.е. поведение нейрона ТC1 не зависит от поведения остальной сети. Когда кривая для неавтономной системы $\left(\right.$ при $\left.k_{\mathrm{NT}} \neq 0\right)$ загибается вниз к кривой автономной системы $\left(k_{\mathrm{NT}}=0\right)$, возбуждение резонансных высокоамплитудных колебаний происходит при меньшем $\bar{a}_{b r}$, поскольку сеть начинает оказывать ответное влияние на ТC1 (конкуренция вынужденных колебаний и автоколебаний в сети). При $k_{\mathrm{NT}}=0.1$ подобное поведение не выражено, поскольку возбуждение вынужденных колебаний происходит практически при том же значении параметра, что и автономных колебаний в сети, а сила воздействия со стороны нейрона NT недостаточна. Далее кривая, соответствующая возбуждению резонансных колебаний, вновь загибается вверх и идет параллельно кривой автономной системы.

Как только возбуждаются высокоамплитудные колебания в ТС1, они передаются на некоторые другие узлы сети, но сеть в целом еще не приходит в возбуждение, поскольку нейроны в сети немного различаются (табл. 1). Вблизи резонанса колебания ТС1 вызывают очень малый отклик в нейроне РY2, на который воздействует ТC1, далее еще меньший в РY3 и далее колебания затухают все сильнее при распространении по сети.
Если рассмотреть самый „удаленный“ по сети от ТС1 нейрон ТС3, колебания в нем практически не могут быть зарегистрированы на доступном уровне точности (рис. 6,d). Поэтому для нейрона ТС3 (рис. 4,b) переход в режим высокоамплитудных колебаний происходит при $\bar{a}_{b r}$ меньшем, чем для ТС1; при этом в отличие от ТC1 порог возбуждения высокоамплитудных колебаний зависит как от $k_{b r}$, так и от $\bar{a}_{b r}$, что естественно, поскольку этот нейрон возбуждается последним во всей сети. Для этого нейрона кривые возбуждения резонансных колебаний сразу идут почти параллельно кривой автономной системы. При этом, однако, колебания в сети остаются многочастотными с различным числом высокоамплитудных импульсов в различных нейронах, как можно видеть из сопоставления рис. $6, c$ и $f$.

Кривые на рис. 4 могут быть построены, поскольку переход от низкоамплитудных колебаний к резонансным происходит резко при очень малом изменении параметра $\bar{a}_{b r}$ (при фиксированном $k_{b r}$ ). Это можно объяснить тем, что, начиная с некоторого значения параметра $\bar{a}_{b r}$, расстройка по частоте, вызванная различиями $a_{\mathrm{NT}}$ и $\bar{a}_{b r}$, уменьшается настолько, что появляется еще одно решение для ТС1, соответствующее высокоамплитудным колебаниям (рис. $6, b$ ). Оно сосуществует с низкоамплитудным решением в небольшой области параметров, как показано на рис. 7 при $k_{\mathrm{NT}}=0.4, k_{b r}=0.6$ - для данных параметров два решения у системы наблюдается в промежутке $\bar{a}_{b r}=1.6125-1.655$. Серая и черная сплошные кривые на рис. 7 показывают эволюцию размаха колебаний нейрона ТC1 при изменении параметра $\bar{a}_{b r}$ в разную сторону (увеличении и уменьшении соответ- 


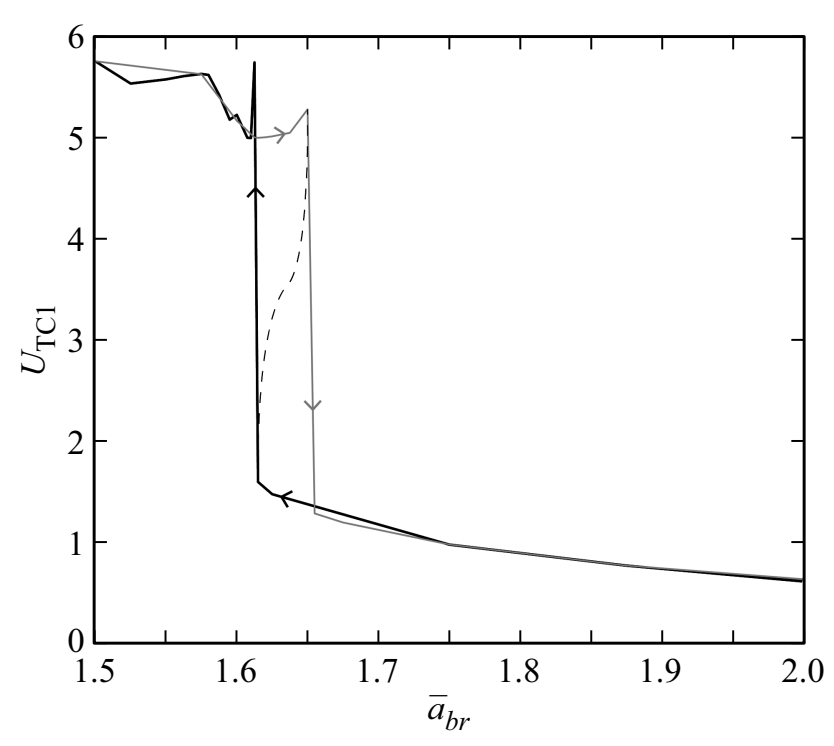

Рис. 7. Резонансная кривая для нейрона TC1 при параметрах $k_{\mathrm{NT}}=0.4, k_{b r}=0.6$. Серым нарисована ветвь, полученная при увеличении $\bar{a}_{b r}$, а черным - ветвь, полученная при уменьшении $\bar{a}_{b r}$.

ственно). В области бистабильности также существует неустойчивый цикл, разделяющий низкоамплитудное и высокоамплитудное решения и схематично показанный на рис. 7 штриховой линией. Это классический случай бистабильности на резонансной кривой, соответствующей нелинейному резонансу, описанный, например, в [25].

\section{3. Генерация эпилептиформной активности}

При инициации эпилептического разряда в таламус из тройничного нерва приходит относительно короткая последовательность импульсов, запускающая колебания в сети. В схеме запуск разряда реализуется за счет кратковременного $(70 \mathrm{~ms})$ открытия ключа $S 1$ через $16 \mathrm{~ms}$ симуляции (рис. 8). Открытый ключ пропускает сигнал внешнего нейрона NT на таламо-кортикальную сеть. Колебания сети сначала носят характер вынужденных, а затем по окончании импульса продолжаются еще некоторое время. По графикам временных рядов (рис. $8, a, c, e, g$ ) видно, что после окончания первичного переходного процесса, соответствующего включению установки, и до подачи внешнего воздействия колебаний в системе нет, а после прекращения внешнего воздействия система продолжает колебаться некоторое время (около 4 периодов). В первые $20 \mathrm{~ms}$ после подачи внешнего воздействия видно, что не все нейроны сети включились в колебания (это переходной процесс, соответствующий установлению вынужденных колебаний), поэтому сигнал нерегулярный и низкоамплитудный. Исходя из фазовых портретов исследованных рядов (рис. $8, b, d, f, h$ ), можно заметить, как система выходит на цикл, соответствующий вынужденным колебаниям, а затем продолжает движение в его окрестности после прекращения воздействия еще некоторое время и только потом скатывается в устойчивую точку.

Возможность продолжения колебаний вызвана тем, что параметр сети можно установить таким образом, чтобы система находилась в подпороговом возбудимом состоянии вблизи бифуркации рождения цикла из сгущения фазовых траекторий. В такой ситуации после прекращения вызванных внешним воздействием колебаний система попадает в сгущение фазовых траекторий [24] и продолжает колебания еще некоторое время. При этом важно геометрическое расположение цикла, соответствующего вынужденным колебаниям, и сгущения траекторий: если они расположены близко, „остаточные“ колебания (обозначим их число $Q$ ) становятся возможны. Фактически такие колебания представляют собой длительный переходной процесс. Они являются в значительной степени регулярными, но в то же время не точно периодичными, что характерно и для экспериментально наблюдаемых эпилептических разрядов. Их амплитуда может отличаться от амплитуды вынужденных колебаний, в том числе даже быть больше нее (рис. 8), что может быть объяснено в том числе ограничением резонанса из-за большой расстройки по частоте между сетью и внешним нейроном NT.

Чтобы можно было сравнить модельные реализации с экспериментальными $[26,27]$, на рис. 8 показаны не колебания отдельных осцилляторов, а временные ряды и фазовые портреты суммарных сигналов групп нейронов, моделирующих разные структуры мозга. Интегральный сигнал групп нейронов PY и IN соответствует сигналу локальных потенциалов первичной соматосенсорной коры (рис. 8, $a, b$ ). Интегральный сигнал группы нейронов RE соответствует сигналу локальных потенциалов ретикулярного ядра таламуса (рис. $8, c, d$ ), а интегральный сигнал группы нейронов ТС - сигналу вентропостериального медиального ядра таламуса (рис. 8,e,f). На рис. 8, $g, h$ показаны временной ряд и фазовый портрет тройничного нерва (NT). При построении рис. 8 были использованы следующие параметры: $\bar{a}_{b r}=1.24125, a_{\mathrm{NT}}=1.025, k_{b r}=0.2, k_{\mathrm{NT}}=0.4$ (близко к кривой $k_{\mathrm{NT}}=0$ на рис. $4, b$ ); время внешнего воздействия $w=70 \mathrm{~ms}$ или около пятнадцати периодов внешнего воздействия. Чтобы пропустить первичный переходной процесс, вызванный изначально разряженными конденсаторами С1 и С2 (рис. 2), воздействие начинается на $16 \mathrm{~ms}$.

Долгий переходной процесс, соответствующий движению на сгущении траекторий после окончания вынужденных колебаний, стоит искать в области между кривой для заданной силы связи внешнего воздействия $k_{\mathrm{NT}}$ и кривой без внешнего воздействия, когда $k_{\mathrm{NT}}=0$, причем требуется достаточно тонкая настройка в пространстве параметров. Например, при уменьшении среднего $\bar{a}_{b r}$ всего на $0.025 \cdot 10^{-3}$ и сохранении других параметров, как на рис. 8, наблюдались только три осцилляции после прекращения воздействия вместо четырех. 

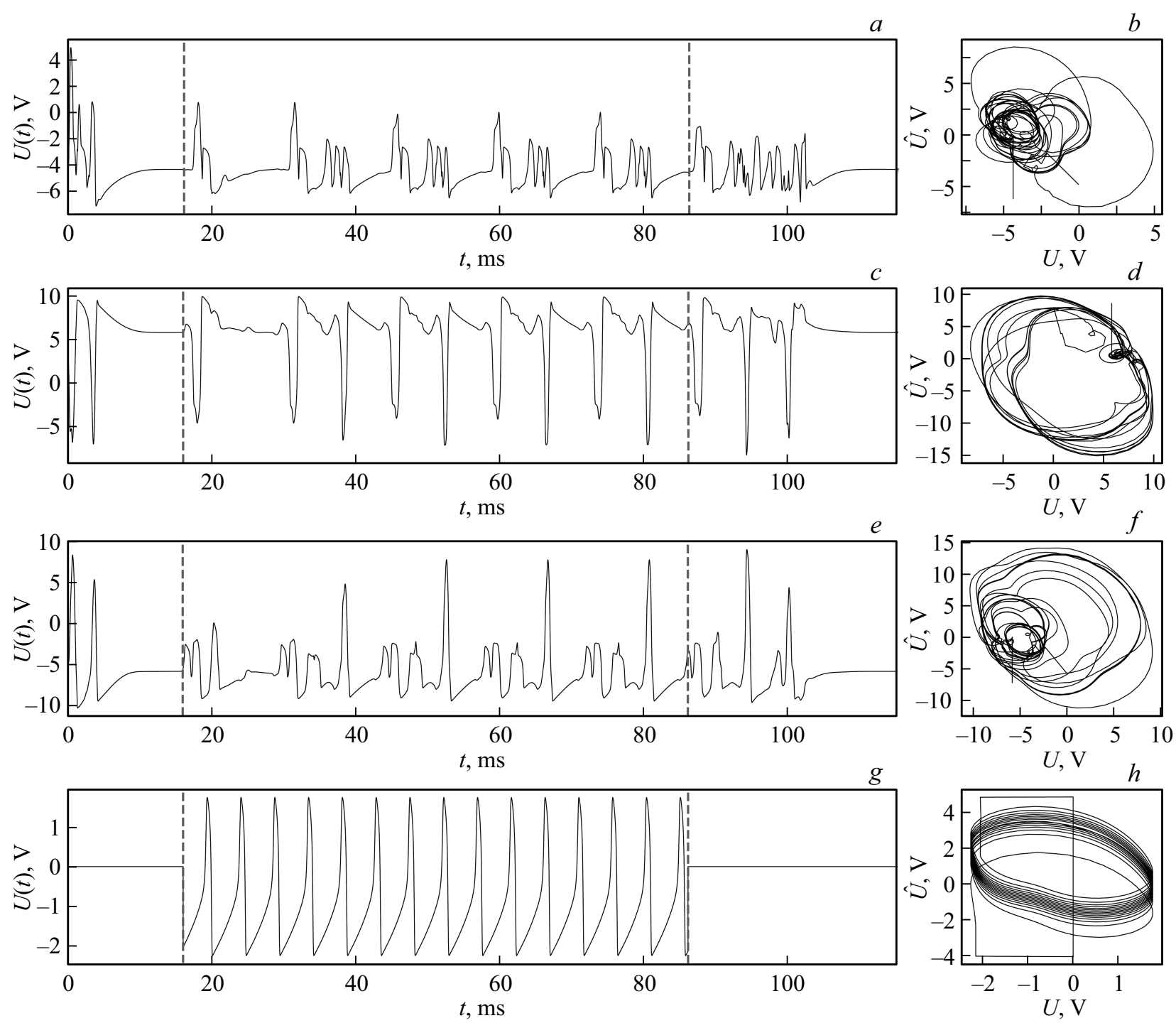

Рис. 8. Временнб́е ряды и фазовые портреты суммарных сигналов генераторов ФитцХью-Нагумо для каждой моделируемой структуры: $a, b$ - первичной соматосенсорной коры $(\mathrm{SI}), c, d-$ ретикулярного ядра таламуса $(\mathrm{RTN}), e, f-$ вентропостериального медиального ядра таламуса, $g, h-$ тройничного нерва (NT).

Таблица 2. Зависимость количества периодов после прекращения воздействия $Q$ от значения порогового параметра $\bar{a}_{b r}$ при фиксированных $k_{b r}=0.2$ и $k_{\mathrm{NT}}=0.4$

\begin{tabular}{c|c|c|c|c|c|c|c|c}
\hline $\bar{a}_{b r}$ & 1.29625 & 1.28375 & 1.27125 & 1.25875 & 1.24625 & 1.24155 & 1.241525 & 1.23375 \\
\hline$Q$ & 0 & 0 & 0 & 1 & 1 & 4 & 6 & $\infty$
\end{tabular}

В табл. 2 показана зависимость количества остаточных колебаний $Q$ от значения порогового параметра системы $\bar{a}_{b r}$ при $k_{b r}=0.2, k_{\mathrm{NT}}=0.4$. Диапазон перебора $\bar{a}_{b r}$ лежит в диапазоне между верхней и нижней штриховой кривой на рис. $4, b$. Видно, что при больших значениях $\bar{a}_{b r}$ ближе к кривой $k_{\mathrm{NT}}=0.4$ колебания в таламо-кортикальной сети прекращаются сразу после окончания внешнего воздействия. Затем в сети наблюдаются по несколько колебаний после окончания воздействия. И довольно быстро при приближении к кривой
$k_{\mathrm{NT}}=0$ колебания не заканчиваются после завершения внешнего воздействия $(Q=\infty)$, что соответствует уже родившемуся из сгущения траекторий циклу.

\section{Заключение}

В работе предложена радиотехническая реализация иерархической нейронной сети - мезамасштабной модели таламо-кортикальной сети мозга. Для предложенной схемы исследованы различные автоколебательные 
режимы и режимы вынужденных колебаний, построены линии бифуркаций, соответствующих рождению цикла (бифуркации Андронова-Хопфа и рождения цикла из сгущения фазовых траекторий) и нелинейному резонансу.

Показано, что предложенная схема может служить моделью возникновения пик-волновых разрядов, характерных для абсансной эпилепсии. Эпилептический разряд инициируется короткой последовательностью импульсов, приходящей от внешнего входа (например, из тройничного нерва) в таламо-кортикальную сеть мозга. В зависимости от параметров сети разряд может не возбуждаться или захватывать не все нейроны в сети (отсутствие генерализации) - в таком случае сеть можно рассматривать как модель нормального функционирования. Если разряд возбуждается, то он представляет собой длинный переходной процесс, состоящий из близких к регулярным колебаний, и заканчивается спонтанно без каких-либо отдельных механизмов прекращения через некоторое время после окончания воздействия, что соответствует современным представлениям об абсансных приступах [28]. При этом схему можно будет в дальнейшем использовать для моделирования эффектов электрофизиологической стимуляции нейронов коры на различных частотах и при различных начальных фазах воздействия; в настоящее время такие эксперименты проводятся только на животных [8,9], что гораздо более затратно и менее удобно. Возбуждение происходит в достаточно узкой области в пространстве параметров, примыкающей к бифуркационной линии, соответствующей бифуркации рождения цикла из сгущения фазовых траекторий. Подобное поведение является исключительно результатом сетевой организации нейронов в модели и не появляется у одного нейрона.

\section{Финансирование работы}

Работа выполнена при поддержке Российского научного фонда, проект № 19-72-10030.

\section{Конфликт интересов}

Авторы заявляют, что у них нет конфликта интересов.

\section{Список литературы}

[1] S. Boccaletti, V. Latora, Y. Moreno, M. Chavez, D.-U.Hwang. Phys. Reports, 424 (4-5), 175 (2006).

[2] О.В. Масленников, В.И. Некоркин. УФН, 187, 745 (2017). [O.V. Maslennikov, V.I. Nekorkin. Phys. Usp., 60, 694 (2017).]

[3] A. Lüttjohann, G. van Luijtelaar. Frontiers in Physiology, 6, 16 (2015).

[4] E.H. Bertram. Epilepsia, 38 (1), 95 (1997).

[5] J.H. Proske, D. Jeanmonod, P.F. Verschure. Europ. J. Neuroscience, 33 (7), 1281 (2011).

[6] W. van Drongelen, H.C. Lee, M. Hereld, Z. Chen, F.P. Elsen, R.L. Stevens. IEEE Transactions on Neural Systems and Rehabilitation Engineer., 13 (2), 236 (2005).
[7] T.M. Medvedeva, M.V. Sysoeva, G. van Luijtelaar, I.V. Sysoev. Neural Networks, 98, 271 (2018).

[8] A. Lüttjohann, G. van Luijtelaar. Epilepsy Research, 106 (12), 136 (2013).

[9] S. van Heukelum, J. Kelderhuis, P. Janssen, G. van Luijtelaar, A. Lüttjohann. Neuroscience, 324, 191 (2016).

[10] B.J. Zhang, M. Chamanzar, M.-R. Alam. J. R. Soc. Interface, 14, 20160872 (2017).

[11] G. Ullah, J.R.Jr. Cressman, E. Barreto, S.L. Schiff. J. Comp. Neuroscience, 26 (2), 171 (2009).

[12] P. Suffczynski, S. Kalitzin, F.H. Lopes da Silva. Neuroscience, 126, 467 (2004).

[13] P.N. Taylor, Y. Wang, M. Goodfellow, J. Dauwels, F. Moeller, U. Stephani. PLoS ONE, 9 (12), e114316 (2014).

[14] G. Paxinos, C. Watson. The rat brain in stereotaxic coordinates. 6th ed. (Academic Press, San Diego, 2006).

[15] A. Coenen, G. van Luijtelaar. Behavioral Genetics, 33 (6), 635 (2003).

[16] C. Marescaux, M. Vergnes, A. Depaulis. J. Neural Transmis. Supplement., 35, 37 (1992).

[17] T.M. Medvedeva, M.V. Sysoeva, I.V. Sysoev. Proceedings of 2nd School on Dynamics of Complex Networks and their Application in Intellectual Robotics. 2018. P. 169.

[18] D. Fan, L. Zhang, Q. Wang. Nonlinear Dynamics, 94, 2807 (2018).

[19] K. Abbasova, S. Chepurnov, N. Chepurnova, G. van Luijtelaar. Brain Research, 1366, 257 (2010).

[20] R. FitzHugh. Bull. Math. Biophys., 17, 257 (1955).

[21] M.A. Dahlem, G. Hiller, A. Panchuk, E. Schöll. Intern. J. Bifurcation Chaos, 19, 745 (2009).

[22] А.С. Дмитричев, Д.В. Касаткин, В.В. Клиньшов, С.Ю. Кириллов, О.В. Масленников, Д.С. Щапин, В.И. Некоркин. Известия вузов. ПНД, 26 (4), 5 (2018). [A.S. Dmitrichev, D.V. Kasatkin, V.V. Klinshov, S.Yu. Kirillov, O.V. Maslennikov, D.S. Shchapin, V.I. Nekorkin. Izvestiya VUZ. Appl. Nonlinear Dynamics, 26 (4), 5 (2018).]

[23] D.D. Kulminskiy, V.I. Ponomarenko, M.D. Prokhorov, A.E. Hramov. Nonlinear Dynamics, 98 (1), 735 (2019).

[24] М.И. Рабинович, Д.И. Трубецков. Введение в теорию колебаний и волн (НИЦ „Регулярная и хаотическая динамика“, 2000)

[25] А.П. Кузнецов, С.П. Кузнецов, Н.М. Рыскин. Нелинейные колебания (Физматлит, М., 2005)

[26] A. Lüttjohann, G. van Luijtelaar. Neurobiology Disease, 47, 47 (2012).

[27] M.V. Sysoeva, A. Lüttjohann, G. van Luijtelaar, I.V. Sysoev. Neuroscience, 314, 75 (2016).

[28] E. Russo, R. Citraro, A. Constanti, A. Leo, A. Lüttjohann, G. van Luijtelaar, G. De Sarro. Neuroscience \& Biobehavioral Rev., 71, 388 (2016). 